\title{
Plasma concentration of Propionibacterium acnes antibodies and prostate cancer risk: results from an Australian population-based case-control study
}

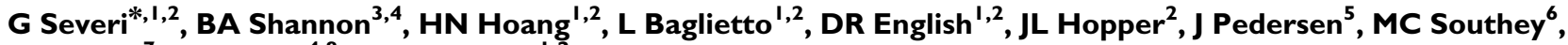 \\ R Sinclair ${ }^{7}$, RJ Cohen ${ }^{4,8}$ and GG Giles ${ }^{1,2}$
}

'Cancer Epidemiology Centre, The Cancer Council of Victoria, Melbourne, Victoria 3053, Australia; ${ }^{2}$ Centre for Molecular, Environmental, Genetic and Analytic Epidemiology, The University of Melbourne, Melbourne, Victoria 3052, Australia; ${ }^{3}$ Tissugen Pty Ltd., Perth, Western Australia 6009, Australia; ${ }^{4}$ School of Pathology and Laboratory Medicine, University of Western Australia, Perth, Western Australia 6009, Australia; ${ }^{5}$ Tissupath Pty Ltd., Melbourne, Victoria 31 22, Australia; ${ }^{6}$ Genetic Epidemiology Laboratory, Department of Pathology, The University of Melbourne, Melbourne, Victoria 3052, Australia; ${ }^{7}$ Department of Dermatology, St Vincent's Hospital, Melbourne, Victoria 3065, Australia; ${ }^{8}$ Uropath Pty Ltd., Perth, Western Australia 6009, Australia

\begin{abstract}
BACKGROUND: Recent studies in prostatic tissue suggest that Propionibacterium acnes ( $P$. acnes), a bacterium associated with acne that normally lives on the skin, is the most prevalent bacterium in the prostate and in men with benign prostatic hyperplasia. Its prevalence is higher in samples from patients subsequently diagnosed with prostate cancer. The aim of our study was to test whether circulating levels of $P$. acnes antibodies are associated with prostate cancer risk and tumour characteristics using plasma samples from a population-based case-control study.

METHODS: We measured plasma concentration of P. acnes antibodies for 809 cases and 584 controls using a recently developed ELISA assay. We compared antibody titres between cases and controls using unconditional logistic regression adjusted for batch and variables associated with the study design (i.e., age, year of selection and centre). The primary analysis included $P$. acnes titres in the model as a dichotomous variable using the median value for controls as the cut-off value.

RESULTS: P. acnes antibody titres for both cases and controls ranged from I : I6 (i.e., low concentration) to I:65 536 (i.e., high concentration; median value $=1$ : 1024). The odds ratio for prostate cancer associated with titres at or above the median value was 0.73 (95\% Cl 0.58-0.9I, P=0.005). The association appeared to be particularly strong for advanced prostate cancer (AJCC Stage grouping III-IV) for which the odds ratio was $0.59(95 \% \mathrm{Cl} 0.43-0.8 \mathrm{I}, P=0.00 \mathrm{I})$ but there was insufficient evidence that the association differed by tumour stage ( $p$ heterogeneity $=0.07$ ).

CONCLUSION: These results need to be confirmed in prospective studies but they are consistent with the hypothesis that $P$. acnes has a role in prostate cancer.

British Journal of Cancer (2010) I 03, 4II-4I5. doi:I0.1038/sj.bjc.6605757 www.bjcancer.com

Published online 6 July 2010

(c) 2010 Cancer Research UK
\end{abstract}

Keywords: prostate cancer; risk factor; acne; P. acnes; case-control study

Studies investigating a possible association between acne and prostate cancer risk have reported inconsistent results. These include a decreased risk associated with facial acne scarring in our Risk Factors for Prostate Cancer Study (RFPCS) (Giles et al, 2003), no significant associations in other two studies (Lightfoot et al, 2004; Galobardes et al, 2005), and an increased risk associated with long-term use of tetracyclines that was assumed to indicate treatment of severe acne (Sutcliffe et al, 2007). Most of these studies (Giles et al, 2003; Lightfoot et al, 2004; Galobardes et al, 2005) were investigating acne as a marker of androgen activity in puberty, under the hypothesis that higher androgen levels might predispose to both acne and development of prostate cancer.

Subsequent research has indicated another possible connection between acne and prostate cancer. Recent reports on Propionibacterium acnes (P. acnes), a bacterium associated with acne that

*Correspondence: Dr G Severi; E-mail: Gianluca.Severi@cancervic.org.au Received 26 January 20 I0; revised I5 April 20I0; accepted I June 20 I0; published online 6 July 2010 normally lives on the skin and thrives in blocked follicles, suggest that this bacterium is prevalent in the prostate, it is associated with acute and chronic prostatic inflammation, and it might have a role in prostate carcinogenesis (Cohen et al, 2005; Alexeyev et al, 2006). Our group (RJC and BAS) cultured $P$. acnes from one-third of a series of radical prostatectomy specimens and found that $P$. acnespositive specimens were more likely to contain foci of acute or chronic inflammation than $P$. acnes-negative specimens (Cohen et al, 2005). Consistent with these findings Alexeyev et al (2006) investigating bacterial DNA in prostatic tissue from patients with benign prostatic hyperplasia found that $P$. acnes was the most prevalent bacterium and that its prevalence was higher in samples from patients subsequently diagnosed with prostate cancer. These observations led to hypothesise that $P$. acnes infection in the prostate gland is positively associated with prostate cancer risk, with acne considered as a marker of increased immune sensitivity to $P$. acnes infection.

The aim of our study was to test whether plasma concentration of $P$. acnes antibodies, measured by an ELISA assay we recently 
developed (Shannon et al, 2008), was associated with prostate cancer risk. We tested this hypothesis using the RFPCS, a population-based case-control study. Although the majority of studies discussed above have suggested positive associations between prostate cancer and either acne in puberty or $P$. acnes infection in the prostate gland, our RFPCS cohort previously showed an inverse association between facial scarring and prostate cancer risk (Giles et al, 2003), therefore a secondary aim of this study was to investigate this apparently conflicting result.

\section{MATERIALS AND METHODS}

\section{Study design and subjects}

The RFPCS study was conducted in 1994-1998 in Melbourne and Perth, Australia. Details of this study were published previously (Giles et al, 2001; Severi et al, 2003). Cases were men aged less than 70 years at diagnosis with histologically confirmed adenocarcinoma of the prostate and notified to the population-based Cancer Registries in the two States during the period between 1994 and 1997. Tumours were staged according to the TNM system and categorised into American Joint Committee on Cancer stage groupings (I-IV) (AJCC, 2002). Cases were classified into two groups according to tumour differentiation: moderate-grade cases (i.e., Gleason score 5-7) and high-grade cases (i.e., Gleason score $8-10)$. Men with well-differentiated tumours or Gleason score less than 5 were excluded. Controls were randomly selected from the state electoral rolls and frequency matched to cases by 5-year age group and city of residence at the time of selection in a ratio of one control per case. Face to face interviews were conducted usually at the men's home using questionnaires to capture various exposures including demographic factors, medical history, anthropometry, radiation exposures, alcohol consumption, smoking, growth and development, medical and surgical procedures related to the reproductive organs, diet captured using a detailed food frequency questionnaire, lifetime history of sexual activity, and family history of prostate cancer. The interview also included questions about acne in adolescence. The interviewer was asked to report 'obvious' acne scarring or 'uncertain' acne scarring (e.g., beard). About mid study, additional funding was obtained to collect blood samples from subjects mainly to extract DNA for genetic association studies and measure levels of markers in circulation. The total number of samples available for this study was 809 for cases and 584 for controls.

\section{Measure of $P$. acnes antibodies}

Anti-P. acnes antibody titres were determined at Tissugen Pty Ltd. (Perth, Western Australia, Australia) by researchers blinded to the case-control status of the plasma samples. The assay was performed as previously described (Shannon et al, 2008), except that incubation with primary and secondary antibodies $(2 \mathrm{~h}$ each, with agitation) and the colour development step $(30 \mathrm{~min})$ were carried out at $37^{\circ} \mathrm{C}$. Briefly, the cell wall-associated/secreted surface proteins harvested from a cultured prostatic $P$. acnes isolate, (type II) were purified, resolubilised to $1.5 \mu \mathrm{g} \mathrm{ml}^{-1}$ in $0.05 \mathrm{M}$ carbonate buffer ( $\mathrm{pH}$ 9.6) and coated overnight onto 96-well ELISA plates. Each plate included no-antigen and no-plasma blanks plus the positive control plasma sample (diluted to $1: 64$ ). Plasma samples were analysed in duplicate using two-fold serial dilutions. Absorbance readings were multiplied by a standardisation factor (the amount required to adjust the positive control to its mean value of 0.957 ) to allow accurate comparison of samples from different runs. The endpoint antibody titre was then determined as the highest serum dilution giving an absorbance reading of 0.100 or greater. The coefficient of intra-assay variation was $5.7 \%$ (as calculated from 18 duplicates of positive control in the same ELISA run) and the inter-assay variation was $6.3 \%$ (as calculated from 18 different ELISA runs of the positive control).

Plasma samples from 69 men were divided into two aliquots to check the replicability of the measures. The laboratory was blind to the duplicates that were included at random among the samples used for the study. As a measure of replicability we used the intraclass correlation coefficient, which is the proportion of the total variance due to variation between persons, where the total variance included a component due to between persons and between measures.

\section{Statistical methods}

The primary hypothesis of association between $P$. acnes antibody titres and prostate cancer risk was tested using unconditional logistic regression with the case-control status as a dependent variable (Breslow and Day, 1980). P. acnes titres were dichotomised using the median value for controls (i.e., 1:1024) and included as independent variable in the logistic model. Odds ratio (OR) estimates and their 95\% confidence intervals (CI) were derived under the likelihood theory. We performed secondary analyses using the tertiles of the distribution of $P$. acnes titres in controls and using the original continuous variable. We $\log _{2}$ transformed the continuous variable before inclusion in the model so that the odds ratio would represent the relative difference in risk associated with a doubling of the titres. We fitted models adjusted for self-reported history of acne during adolescence or facial acne scarring to investigate whether this would affect the association between $P$. acnes titres and prostate cancer risk. We also tested the interaction between $P$. acnes titres and prostate cancer risk and self-reported history of acne in adolescence, facial acne scarring and reference age (age at diagnosis for cases and age at selection for controls). Odd ratios by tumour stage (stage I-II and stage III-IV) and grade (moderate-grade and high-grade) were estimated using polytomous logistic regression models. All regression analyses were adjusted for batch and for variables associated with the original study design: reference age $(<55,55-59,60-69)$, study centre (Melbourne and Perth), selection year (1994, 1995, 1996, and 1997). Further adjustment for family history of prostate cancer (i.e., number of first-degree relatives affected), country of birth (Australia $v s$ others), and self-reported history of benign prostatic hyperplasia did not materially change the estimates. We tested whether adult $P$. acnes antibody titres were associated with self-reported acne during adolescence or facial acne scarring by applying linear regression to the logarithm of the inverse of the titres for controls using history of acne or facial scarring as predictors. We tested for trend in the odds ratios by comparing the likelihoods of two models with and without a pseudo-continuous variable that was constructed first by $\log _{2}$ transforming the original variable and then by assigning each man in a specific tertile, the corresponding median value for that tertile. We tested hypotheses using the likelihood ratio test. All tests were two-sided and nominal statistical significance was based on $P<0.05$. All statistical analyses were performed using Stata/SE 10 (Stata Corporation, College Station, Texas, USA).

\section{RESULTS}

We measured $P$. acnes antibody titres for a total of 809 cases and 584 controls whose characteristics are described in Table 1. There was little variation between the duplicates as indicated by the high intra-class correlation coefficient $(0.94 ; 95 \%$ CI $0.91-0.97)$. $P$. acnes antibody titres measured for RFPCS participants ranged from $1: 16$ (i.e., low concentration) to $1: 65536$ (i.e., high concentration) and the median value was $1: 1024$. The mean $\log _{2}$ titres for controls with and without a history of acne were 10.6 and 9.8 , respectively. The analysis of $P$. acnes titres for controls showed 
that after adjusting for the study variables titres for men reporting a history of acne in adolescence were $85 \%$ higher than titres for those not reporting a history of acne $(P=0.003)$. We found similar results for facial acne scarring (data not shown).

The distribution of $P$. acnes antibody titres is presented separately for cases and controls in Figure 1. The median and range of the titres were the same for cases and controls but samples with high titres (e.g., 1:2048 or more) appeared to be more prevalent for controls than for cases. The mean $\log _{2}$ titres for controls and cases were 10 and 9.7, respectively. When we fitted a logistic regression model to the titres split in two groups using the median as cut-off point $(1: 1024)$, the odds ratio associated with titres equal to or above the median compared with titres below the median was $0.73(95 \%$ CI $0.58-0.91)$ (Table 2). The odds ratio was 0.59 (95\% CI $0.43-0.81)$ for advanced prostate cancer (stage III-IV) and $0.79(95 \%$ CI $0.62-1.01)$ for early-stage prostate cancer (stage I-II), but there was insufficient evidence to conclude that the association differs by tumour stage (p-heterogeneity $=0.07)$. The odds ratios for moderate-grade prostate cancer were very similar to the odds ratios for high-grade prostate

Table I Characteristics of participants in the Australian Risk Factors for Prostate Cancer Study

\begin{tabular}{lcc}
\hline Factor & Cases (\%) $\mathbf{N}=\mathbf{8 0 9}^{\mathbf{a}}$ & Controls (\%) $\mathbf{N}=\mathbf{5 8 4}^{\mathbf{a}}$ \\
\hline Reference age & & \\
$<55$ years & $113(14)$ & $111(19)$ \\
$55-59$ years & $194(24)$ & $98(17)$ \\
$60-69$ years & $502(62)$ & $375(64)$ \\
Country of birth & & \\
$\quad$ Australia & $567(70)$ & $383(66)$ \\
Overseas & $241(30)$ & $200(34)$ \\
Self-reported history of acne in adolescence & \\
No & $641(79)$ & $456(78)$ \\
Yes & $167(21)$ & \\
Facial acne scarring & & \\
Not obvious & $735(91)$ & $518(89)$ \\
Obvious & $27(3)$ & $28(5)$ \\
Uncertain (beard) & $35(4)$ & $37(6)$ \\
\hline
\end{tabular}

The number of missing values for cases and controls were: I and I for country of birth; 1 and 3 for self-reported history of acne in adolescence; 12 and 1 for facial acne scarring. cancer $(p$-heterogeneity $=0.8)$. The association between $P$. acnes antibody titres was confirmed when we fitted the logistic regression including the titres as continuous variables. The odd ratio for doubling the antibody titres was 0.94 (95\% CI $0.90-0.99$ ). In the analysis by tertiles of the distribution of $P$. acnes titres in controls, the odds ratios associated with the second and third tertiles compared with the first tertile were 1.00 (95\% CI $0.76-1.34)$ and 0.80 (95\% CI $0.60-1.06)$, respectively (test for linear trend, $P=0.06$ ).

The odds ratios for $P$. acnes titres did not materially change after the inclusion of self-reported history of acne or facial acne scarring in the models (data not shown), indicating that the association between $P$. acnes antibody titres and prostate cancer risk is independent of self-reported history of acne during adolescence or facial acne scarring.

For self-reported history of acne during adolescence the odds ratio for overall prostate cancer was 0.93 (95\% CI $0.70-1.22)$. For facial acne scarring, the odds ratios for overall prostate cancer was 0.66 (95\% CI $0.37-1.17$ ) for the obvious compared with the not obvious. We found no statistically significant evidence of interactions between $P$. acnes antibody titres and self-reported history of acne during adolescence or $P$. acnes antibody titres and facial acne scarring after removing men with uncertain acne scarring.

\section{DISCUSSION}

The findings from our case-control study suggest that higher concentrations of circulating $P$. acnes antibodies measured in adulthood are associated with decreased risk of prostate cancer, especially for advanced prostate cancer. The strengths of this study include the excellent repeatability of the plasma measures, the population-based design, and the relatively large sample size. One limitation is the lack of specificity of the $P$. acnes antibodies for subtypes prevalent in the prostate. Although the ELISA was developed from isolates cultured from the prostate, immunoblots showed that antigens were conserved among other types of $P$. acnes and thus our assay could not differentiate between immune responses raised against $P$. acnes infection in the prostate and $P$. acnes infection in the skin or in other organs. In our retrospective study blood samples for cases were collected after diagnosis and we cannot rule out that the antibody titres in our prostate cancer cases were lowered by the tumour itself or by androgen-deprivation therapy. However, this is unlikely because cancer tends to upregulate the antibody-mediated immune response (Filella et al, 2000; Johansson et al, 2008) and evidence

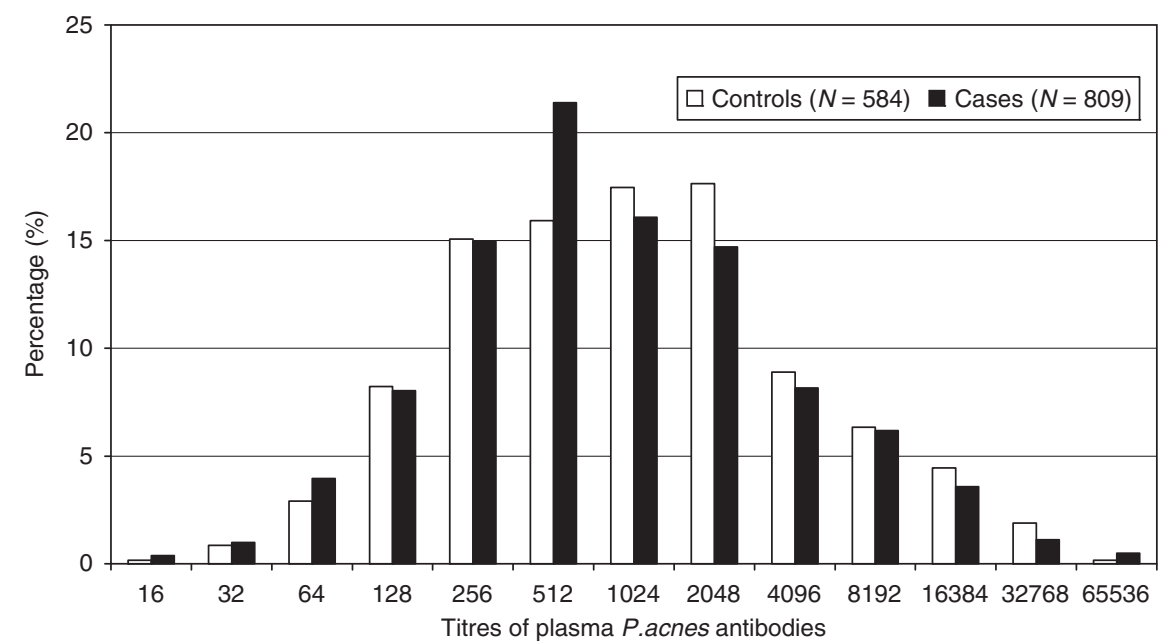

Figure I Distribution of P.acnes antibody titres for prostate cancer cases and controls from the Australian Risk Factors for Prostate Cancer Study. 
414 suggests that androgen deprivation therapy may increase, rather than decrease, immune function and antibody production (Aragon-Ching et al, 2007).

Since $P$. acnes induces a strong cell-mediated T-helper type 1 (Th1) immune response (Mouser et al, 2003; Namazi, 2007) a more likely explanation of our findings is that higher antibody titres are an indirect marker of increased cell-mediated immunity caused by $P$. acnes infection and that this increased immunity protects from prostate cancer.

Persistent exposure to Th1 cytokines through chronic infection can result in systemic Th1 polarisation of the immune system (Romagnani, 2004), therefore, people with a history of acne or $P$. acnes-related infection of the prostate gland could be predisposed to develop a strong systemic Th1-type immune response. Th1 immune responses are detrimental to the establishment and progression of tumours, which typically secrete factors to suppress Th1 and promote T-helper type 2 (humoral or antibody-mediated) immunity (Johansson et al, 2008). In prostate cancer patients this switch to Th2 polarisation occurs in association with development of metastatic disease (Filella et al, 2000), possibly explaining why the inverse association between $P$. acnes titres and prostate cancer risk would be stronger for advanced-stage disease.

Several lines of evidence support the possible involvement of immune responses against $P$. acnes in protection against prostate cancer. First, $P$. acnes is a potent immunomodulator that has been trialed as a cancer treatment in both animals and humans. The tumouricidal activity of $P$. acnes is mediated through recruitment and activation of innate immune cells including macrophages and natural killer T cells (Ananias et al, 2007). Second, acne has been indicated as a protective factor against various other disorders associated with a Th2 immune response, including melanomas, lymphomas, leukaemias, atopy, allergy, and asthma (Namazi, 2007). Finally, the inflammation commonly observed in the prostate gland is predominantly composed of Th1 lymphocytes and macrophages, which indicates a delayed-type hypersensitivity (Th1-type) immune response (McClinton et al, 1990; Sfanos et al, 2008).

As our study collected blood samples from adult men, we cannot distinguish between high titres established by acne during puberty and those arising later in response to prostate infection. Involvement of immunity levels maintained since puberty may be possible since previous research by Ingham et al (1987) has shown that antibody titres against $P$. acnes are established in early puberty and still exist at similar levels in individuals 65 years old. In that study the mean $\log _{2}$ titres were slightly higher than the ones we found in our case-control study, but the absolute values cannot be compared directly due to differences in assay sensitivity. However, since we observed that higher anti- $P$. acnes antibody titres were inversely associated with prostate cancer risk independently of a history of acne during adolescence, it is possible that in some men higher titres indicate infection of the prostate gland.

While this study supports our previous finding of an inverse association between facial acne scarring and prostate cancer risk in the RFPCS study (Giles et al, 2003), it is difficult to reconcile this observed inverse association with our previous finding that $P$. acnes culture-positive radical prostatectomy specimens were more likely to contain foci of acute or chronic inflammation (Cohen et al, 2005). In that study, however, it was not possible to investigate the correlation between the inflammatory immune response in the prostate with antibody levels in circulation because serum was not available.

In conclusion, the inverse association that we have observed between concentrations of circulating $P$. acnes antibodies and risk of prostate cancer needs to be replicated in large prospective studies to exclude the possibility that the increased concentrations in cases are caused by the tumour itself or by treatment. If our finding is confirmed it might open an avenue for the prevention of this disease. 


\section{ACKNOWLEDGEMENTS}

We thank the study participants, and the many urologists, nurses, and histopathologists who kindly facilitated the recruitment and collection of patient information and pathologist reports. We thank Shang-Chih (Sun) Chen for his excellent work in performing the ELISA tests. This

\section{REFERENCES}

AJCC (2002) Prostate. In American Joint Committee on Cancer: AJCC Cancer Staging Manual. 6th edn, pp 309-316. Springer: New York

Alexeyev O, Bergh J, Marklund I, Thellenberg-Karlsson C, Wiklund F, Gronberg H, Bergh A, Elgh F (2006) Association between the presence of bacterial 16S RNA in prostate specimens taken during transurethral resection of prostate and subsequent risk of prostate cancer (Sweden). Cancer Causes Control 17: 1127-1133

Ananias RZ, Rodrigues EG, Braga EG, Squaiella CC, Mussalem JS, Longhini AL, Travassos LR, Longo-Maugeri IM (2007) Modulatory effect of killed Propionibacterium acnes and its purified soluble polysaccharide on peritoneal exudate cells from $\mathrm{C} 57 \mathrm{Bl} / 6$ mice: major NKT cell recruitment and increased cytotoxicity. Scand J Immunol 65: 538-548

Aragon-Ching JB, Williams KM, Gulley JL (2007) Impact of androgendeprivation therapy on the immune system: implications for combination therapy of prostate cancer. Front Biosci 12: 4957 - 4971

Breslow N, Day N (1980) Statistical Methods in Cancer Research. Vol I. The Analysis of Case-Control Studies. International Agency for Research on Cancer: Lyon, France

Cohen RJ, Shannon BA, McNeal JE, Shannon T, Garrett KL (2005) Propionibacterium acnes associated with inflammation in radical prostatectomy specimens: a possible link to cancer evolution? J Urol 173: $1969-1974$

Filella X, Alcover J, Zarco MA, Beardo P, Molina R, Ballesta AM (2000) Analysis of type T1 and T2 cytokines in patients with prostate cancer. Prostate 44: $271-274$

Galobardes B, Davey Smith G, Jeffreys M, Kinra S, McCarron P (2005) Acne in adolescence and cause-specific mortality: lower coronary heart disease but higher prostate cancer mortality: the Glasgow Alumni Cohort Study. Am J Epidemiol 161: 1094-1101

Giles GG, Severi G, English DR, McCredie MR, MacInnis R, Boyle P, Hopper JL (2003) Early growth, adult body size and prostate cancer risk. Int J Cancer 103: $241-245$

Giles GG, Severi G, McCredie MR, English DR, Johnson W, Hopper JL, Boyle P (2001) Smoking and prostate cancer: findings from an Australian case-control study. Ann Oncol 12: 761-765 study was supported by infrastructure from the Cancer Council Victoria, and grants from the National Health and Medical Research Council of Australia (no. 450104, no. 504700, and no. 504702), the Tattersall's, The Whitten Foundation, and the Prostate Cancer Foundation of Australia. The study was approved by the Ethics Review Committee of the Cancer Council of Victoria (reference no. HREC 9500).

ngham E, Gowland G, Ward RM, Holland KT, Cunliffe WJ (1987) Antibodies to P. acnes and P. acnes exocellular enzymes in the normal population at various ages and in patients with acne vulgaris. Br J Dermatol 116: 805-812

Johansson M, Denardo DG, Coussens LM (2008) Polarized immune responses differentially regulate cancer development. Immunol Rev 222: $145-154$

Lightfoot N, Conlon M, Kreiger N, Sass-Kortsak A, Purdham J, Darlington G (2004) Medical history, sexual and maturational factors and prostate cancer risk. Ann Epidemiol 14: 655-662

McClinton S, Eremin O, Miller ID (1990) Inflammatory infiltrate in prostatic hyperplasia-evidence of a host response to intraprostatic spermatozoa? Br J Urol 65: 606-610

Mouser PE, Baker BS, Seaton ED, Chu AC (2003) Propionibacterium acnes-reactive $\mathrm{T}$ helper-1 cells in the skin of patients with acne vulgaris. J Invest Dermatol 121: 1226-1228

Namazi MR (2007) Extension of the 'Hygiene Hypothesis' to the negative association between acne and atopy, hematological malignancies, and malignant melanoma. Med Hypotheses 69: 960 - 961

Romagnani S (2004) The increased prevalence of allergy and the hygiene hypothesis: missing immune deviation, reduced immune suppression, or both? Immunology 112: $352-363$

Severi G, Giles GG, Southey MC, Tesoriero A, Tilley W, Neufing P, Morris H, English DR, McCredie MR, Boyle P, Hopper JL (2003) ELAC2/HPC2 polymorphisms, prostate-specific antigen levels, and prostate cancer. J Natl Cancer Inst 95: 818-824

Sfanos KS, Bruno TC, Maris CH, Xu L, Thoburn CJ, DeMarzo AM, Meeker AK, Isaacs WB, Drake CG (2008) Phenotypic analysis of prostateinfiltrating lymphocytes reveals TH17 and Treg skewing. Clin Cancer Res 14: $3254-3261$

Shannon BA, Cohen RJ, Garrett KL (2008) The antibody response to Propionibacterium acnes is an independent predictor of serum prostatespecific antigen levels in biopsy-negative men. BJU Int 101: 429-435

Sutcliffe S, Giovannucci E, Isaacs WB, Willett WC, Platz EA (2007) Acne and risk of prostate cancer. Int J Cancer 121: 2688-2692 